\title{
AN ON-LINE METHOD FOR THERMAL DIFFUSIVITY DETECTION OF THIN FILMS USING INFRARED VIDEO
}

\author{
Huilong Dong, Boyu Zheng, Feifan Chen
}

Tsinghua University, Department of Precision Instrument, Beijing, 10084, China (donghl11@mails.tsinghua.edu.cn, zhengby12@mails.tsinghua.edu.cn, $\bowtie$ cff@mail.tsinghua.edu.cn, +86 1062796281 )

\begin{abstract}
A novel method for thermal diffusivity evolution of thin-film materials with pulsed Gaussian beam and infrared video is reported. Compared with common pulse methods performed in specialized labs, the proposed method implements a rapid on-line measurement without producing the off-centre detection error. Through mathematical deduction of the original heat conduction model, it is discovered that the area $s$, which is encircled by the maximum temperature curve $r_{T M A X}(\theta)$, increases linearly over elapsed time. The thermal diffusivity is acquired from the growth rate of the area $s$. In this study, the off-centre detection error is avoided by performing the distance regularized level set evolution formulation. The area $s$ was extracted from the binary images of temperature variation rate, without inducing errors from determination of the heat source centre. Thermal diffusivities of three materials, 304 stainless steel, titanium, and zirconium have been measured with the established on-line detection system, and the measurement errors are: $-2.26 \%,-1.07 \%$, and $1.61 \%$ respectively.
\end{abstract}

Keywords: thermal diffusivity, on-line detection, off-centre error, infrared video, thin films.

(C) 2016 Polish Academy of Sciences. All rights reserved

\section{Introduction}

In recent years, varieties of thin-film materials have been widely used as the key components in Micro-Electromechanical Systems (MEMS) [1-2], biotechnology [3], microfluidics [4-5], etc. A knowledge of these thin-film materials' thermal diffusivity is essential to monitor the thermal diffusion process and solve some engineering application problems, such as heat dissipation of MEMS devices. There are some methods for thermal diffusivity estimation, among which the pulse methods are widely applied for their rapidity and simplicity. Specifically, as a typical pulse method, the flash method [6] is widely used for evaluation of the axial thermal diffusivity. As a development of the flash technique, the converging thermal wave technique [7] is used for the radial thermal diffusivity measurement by monitoring the temperature evolutions of the annular heat source centre. Besides, Cernuschi et al. determined the radial thermal diffusivity evolution by monitoring the temperature distribution along a line crossing the heat source centre [8].

For all the typical pulse methods mentioned above, the thermal diffusivity was measured by analyzing the temperature evolutions of one or a few points on a sample surface. Moreover, an accurate localization of the heat source centre position is required for calculation of the thermal diffusivity [7-14]. Therefore, the thermal diffusivity measurements are usually performed in specialized labs using a complex measurement system with precisely located optical devices.

On the other hand, infrared thermography technology has been developing rapidly in recent years. It enables estimation of the average thermal diffusion characteristics by analyzing infrared thermography of the whole thermal diffusing area, instead of numerous points. In this paper, a new method using instantaneous Gaussian laser beam irradiating and an IR camera 
detecting the temperature field is proposed. This method is advantageous for its capability of implementing a simple and fast on-line measurement avoiding the off-centre detection error. The average thermal diffusion characteristics is obtained by monitoring the growth rate of the area $s$ encircled by the maximum temperature curve $r_{T M A X}(\theta)$. To avoid the off-centre detection error, the Distance Regularized Level Set Evolution (DRLSE) [15] formulation is performed to calculate the area $s$ without determining the heat source centre. Based on the above features, a simple on-line detection system can be quickly constructed.

\section{Principle}

\subsection{Heat transfer model}

Figure 1 describes a three-dimensional heat transfer model. When a Gaussian laser beam with the radius of $R_{c}$ instantaneously heats a plate-like isotropic sample, the temperature evolution on the sample surface can be described by a three-dimensional differential function in the cylindrical coordinate system:

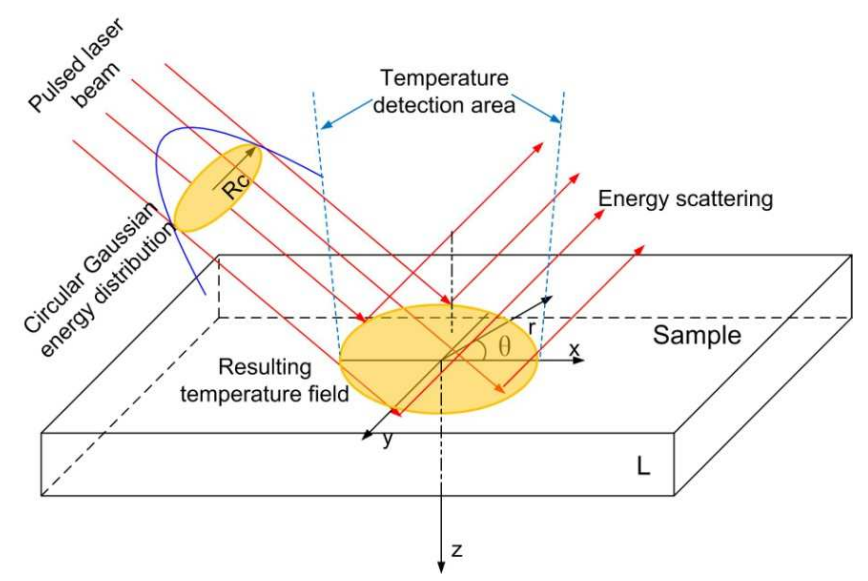

Fig. 1. A schematic of the heat transfer model.

$$
\begin{gathered}
\frac{1}{\alpha} \frac{\partial T(r, \theta, z, t)}{\partial t}=\frac{1}{r} \frac{\partial}{\partial r}\left(r \frac{\partial T(r, \theta, z, t)}{\partial r}\right)+\frac{1}{r^{2}} \frac{\partial}{\partial \theta}\left(\frac{\partial T(r, \theta, z, t)}{\partial \theta}\right)+\frac{\partial}{\partial z}\left(\frac{\partial T(r, \theta, z, t)}{\partial z}\right), \\
-\alpha \rho c \frac{\partial T(r, \theta, 0, t)}{\partial z}=\frac{2 Q}{\pi R_{c}^{2}} e^{-2 r^{2} / R_{c}^{2}} \delta(t), \\
-\alpha \rho c \frac{\partial T(r, \theta, L, t)}{\partial z}=0,
\end{gathered}
$$

where: $\rho$ is the density; $T$ is the temperature; $\alpha$ is the thermal diffusivity; $L$ is the sample thickness; $c$ is the specific heat; $Q$ is the energy of the pulsed laser beam; $t, r, \theta$ and $z$ are the time, radial distance, polar angle and height, respectively. $\delta(t)$ is the Dirac function. (2) shows the boundary condition of the front surface. (3) shows the boundary condition of the rear surface under the assumption of heat insulation. (1) has been solved combined with (2) and (3) as follows [8]: 
where:

$$
T(r, z, t)=Y(z, t) \frac{1}{R_{c}^{2}+8 \alpha t} \exp \left(-\frac{2 r^{2}}{R_{c}^{2}+8 \alpha t}\right),
$$

$$
Y(z, t)=\frac{2 Q}{\rho c \sqrt{\alpha \pi^{3} t}} \sum_{n=-\infty}^{+\infty} \exp \left\{-\frac{[(n-1) L+z / 2]^{2}}{\alpha t}\right\} .
$$

When $L$ is in order of micron or submillimetre, $\partial Y(z, t) / \partial t \approx 0$ can be obtained. Therefore, the function $Y(z, t)$ can be approximately considered as a unary function $Y(z)$. Thus, the function $T(r, z, t)$ can be simplified as:

$$
T(r, z, t)=Y(\mathrm{z}) \frac{1}{R_{c}^{2}+8 \alpha t} \exp \left(-\frac{2 r^{2}}{R_{c}^{2}+8 \alpha t}\right) .
$$

Setting $\partial T(r, z, t) / \partial t=0$, the following equation can be obtained for $t>0$ :

$$
r_{T M A X}^{2}(\theta)=4 \alpha t+R_{c}^{2} / 2,
$$

where $\operatorname{rTMX}_{\text {TMA }}(\theta)$ is the maximum temperature curve. Fig. 2 is introduced here to explain (7). Figs. 2a, 2b, and 2c show the computed $r_{T M A X}(\theta)$ (red curves) at $t=0.5 \mathrm{~s}, t=0.75 \mathrm{~s}$, and $t=1 \mathrm{~s}$, respectively. The function $r_{T M A X}(\theta)$ is computed by fixing $\alpha=9.32 \times 10^{-6} \mathrm{~m}^{2} \mathrm{~s}^{-1}$ and $R_{c}=0.5$ $\mathrm{mm}$. At a specific time $t=t_{0}$, the temperature is falling $(d T / d t<0)$ in the closed red curve, and rising $(d T / d t>0)$ outside. Particularly, the temperature rises to the maximum $(d T / d t=0)$ on the red curve. Also, the area $s$ encircled by the red curve $r_{T M A X}(\theta)$ grows with time increasing.
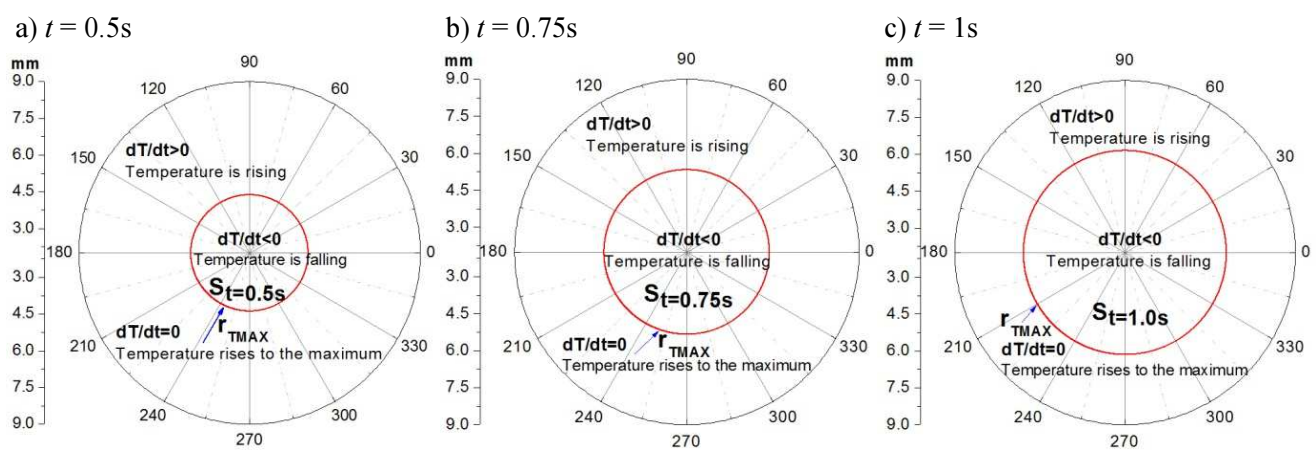

Fig. 2. Curves of $r_{T M A X}(\theta)$ computed by (7).

Let us calculate the definite integral for (7):

$$
\begin{gathered}
\int_{0}^{2 \pi} r_{T M A X}^{2}(\theta) d \theta=\int_{0}^{2 \pi} R_{c}^{2} / 2 d \theta+\int_{0}^{2 \pi} 4 \alpha t d \theta, \\
s=0.5 \pi R_{c}^{2}+4 \pi \alpha t,
\end{gathered}
$$

where $s$ is the area encircled by $r_{\text {TMAX }}(\theta)$, as shown in Fig. 2. (9) shows that $s$ increases linearly over elapsed time, and the thermal diffusivity $\alpha$ can be obtained from the growth rate. 


\subsection{Thermal diffusivity measurement}

The following section discusses the detailed process of thermal diffusivity characterization. Using the IR camera as the temperature detector, a sequence of thermal images can be obtained. Every pixel in the image indicates an actual temperature detection point. In particular, the temperature evolutions of all the $n \times m$ pixels in the IR image are extracted:

$$
T_{O}(t)=\left[\begin{array}{cccc}
T_{O 11}(t) & T_{O 12}(t) & \ldots & T_{O 1 m}(t) \\
T_{O 21}(t) & \ddots & & \vdots \\
\vdots & & T_{O i j}(t) & \vdots \\
T_{O n 1}(t) & \ldots & \ldots & T_{O n m}(t)
\end{array}\right]_{n \times m},
$$

where $T_{O i j}(t)$ is the temperature evolution of the pixel in $j$-th column and $i$-th row of the image. To reduce thermal noise, the following polynomial approximation is applied:

$$
T_{P i j}(t)=b_{N} t^{N}+b_{N-1} t^{N-1}+\cdots+b_{1} t+b_{0},
$$

where $T_{P i j}(t)$ is the polynomial approximation of $T_{O}{ }_{i j}(t)$. The temperature variation rate can then be calculated:

$$
\frac{d T_{p}(t)}{d t}=\left[\begin{array}{cccc}
d T_{P 11}(t) / d t & d T_{P 12}(t) / d t & \ldots & d T_{P 1 m}(t) / d t \\
d T_{P 21}(t) / d t & \ddots & & \vdots \\
\vdots & & d T_{P i j}(t) / d t & \vdots \\
d T_{P n 1}(t) / d t & \ldots & \ldots & d T_{P n m}(t) / d t
\end{array}\right]_{n \times m}
$$

Then, the following binarization processing is done:

$$
d T_{P i j}(t) / d t=\left\{\begin{array}{ll}
\text { TFALL } & d T_{P_{i j}}(t) / d t<0 \\
\text { TRISE } & d T_{P i j}(t) / d t \geq 0
\end{array} .\right.
$$

TFALL and TRISE are constants indicating the temperature fall and rise, respectively. Substituting (13) into (12), the binarization processed images of temperature variation rate are obtained. As described in Fig. 2, the binary images of temperature variation rate have the Temperature Falling Region (TFALL) inside the maximum temperature curve $r_{T M A X}(\theta)$, whereas the Temperature Rising Region (TRISE) outside it. The maximum temperature curve $r_{T M A X}(\theta)$ is located between the two regions.

Then, introducing the coefficient $e$ :

$$
e=4 \pi \alpha
$$

Equation (9) can be written as:

$$
s=0.5 \pi R_{c}^{2}+e t .
$$

The area $s$ encircled by $r_{T M A X}(\theta)$ is calculated from the binary image of temperature variation rate. A sequence of $\left(s_{1}, t_{1}\right) \sim\left(s_{\mathrm{k}}, t_{\mathrm{k}}\right)$ is extracted to perform a linear regression combined with (15). The thermal diffusivity $\alpha$ is calculated by the slop $e$ of the regression line:

$$
\alpha=e / 4 \pi \text {. }
$$


In addition, to calculate $s$ accurately, $r_{T M A X}(\theta)$ is computed intelligently by performing image segmentation in the binary images of temperature variation rate. In particular, the DRLSE formulation has been demonstrated effective in avoiding the induced numerical errors and reducing the number of iterations for the image segmentation of edge-based active contour models [15-19]. Therefore, in this paper the DRLSE formulation is introduced to calculate $r_{\text {TMAX }}(\theta)$.

Figure 3 shows the detailed thermal diffusivity characterization process. It can be summarized as:

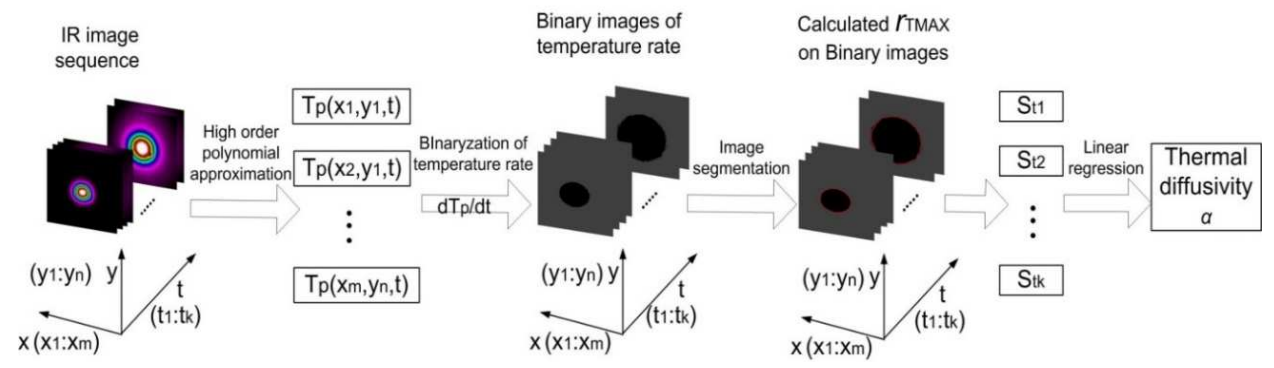

Fig. 3. The thermal diffusivity characterization process.

1) Perform polynomial approximation to obtain $T_{P 11}(t) \sim T_{P n m}(t)$.

2) Calculate $d T_{P}(t) / d t$ and carry out binarization processing using (13).

3) Perform DRLSE formulation to calculate $r_{T M A X}(\theta)$ in binary images of temperature variation rate.

4) Perform linear regression by inserting $\left(s_{1}, t_{1}\right) \sim\left(s_{\mathrm{k}}, t_{\mathrm{k}}\right)$ into (15).

5) Calculate the thermal diffusivity $\alpha$ using (16).

\section{On-line detection system}

Figure 4 shows the on-line measurement system configuration. A measured thin film structure is fixed on the workpiece. The semiconductor laser instantaneously heats the measurement surface. The IR camera is used as the temperature detector. The Personal Computer (PC) performs real-time temperature recording and online calculation.

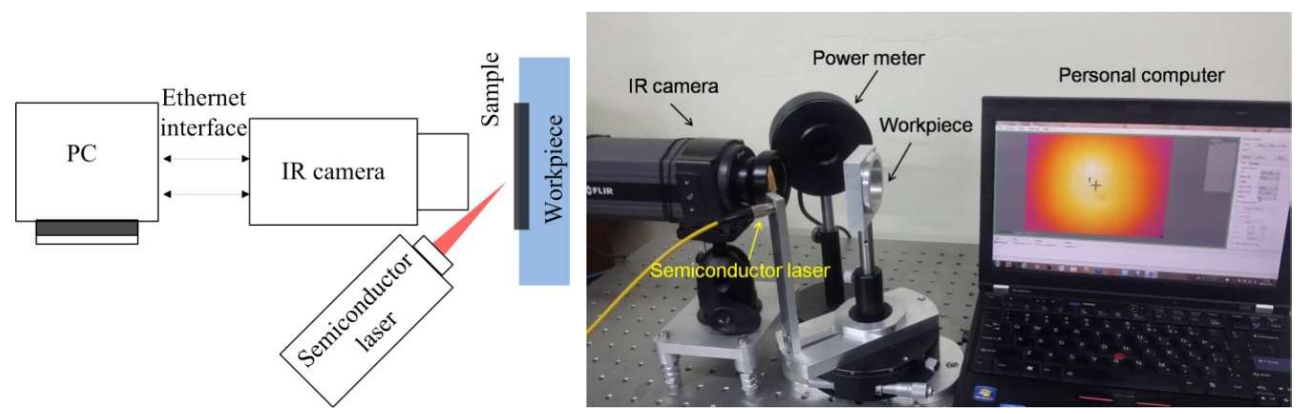

Fig. 4. The on-line measurement system for thermal diffusivity detection.

The semiconductor laser (Type: BWT DS2-50.00 W) with the pulse mode is used. A pulsed Gaussian beam is focused by fixing a self-focusing lens on the optical fibre head. The radius of the Gaussian beam on the sample surface is roughly $0.5 \mathrm{~mm}$. The laser power and the pulse 
width are $25 \mathrm{~W}$ and $62.5 \mathrm{~ms}$, respectively. The energy finally absorbed by the materials can be calculated to be lower than $0.3125 \mathrm{~J}$ due to a high sample surface reflectivity of about $80 \%$ (It is determined by a laser power meter (Type: WG-LP-3C-50.00 W) with measuring range of $50 \mathrm{~W}$ and reading accuracy of $0.1 \mathrm{~W}$, placed in the reflection direction as shown in Fig. 4).

The IR camera (Type: FLIR A315), with the thermal sensitivity better than $50 \mathrm{mK}$ and the spectral range of $7.5 \mu \mathrm{m}$ to $13 \mu \mathrm{m}$, can detect the object temperature within the range of $273 \mathrm{~K}$ to $623 \mathrm{~K}$. The IR image contains $240 \times 320$ pixels, and the actual distance between two neighbouring pixels is calculated to be roughly $98 \mu \mathrm{m}$. The PC records the real-time infrared videos through an Ethernet interface with the video frequency of $60 \mathrm{~Hz}$. The temperature detection accuracy and emissivity are not the key factors, because only the relative variation of temperature is required.

The thin-film samples of 304 stainless steel, titanium, and zirconium with thickness of $200 \mu \mathrm{m}$ and diameter of $90 \mathrm{~mm}$ have been prepared for validation of the method.

\section{Results and discussion}

\subsection{Temperature information extraction}

Detection experiments have been performed. Altogether 120 infrared images $(240 \times 320)$ have been recorded immediately after the heat excitation within the time range of $0<t<2 \mathrm{~s}$. From the recorded infrared images, the temperature evolutions of $76800(240 \times 320)$ positions $T_{011}(t) \sim T_{0240320}(t)$ on the sample surface could then be extracted using (10).

Figures $5 \mathrm{a}$ and $5 \mathrm{~b}$ show the temperature evolutions (black curves) $T_{0}(r=3 \mathrm{~mm}, t)$, $T_{0}(r=4 \mathrm{~mm}, t)$, and $T_{0}(r=5 \mathrm{~mm}, t)$, and the corresponding polynomial approximation results (red curves), for titanium and zirconium samples, respectively. The heat excitation source centre is set as the cylindrical coordinate system origin $(r=0)$, then the radius distance $r$ indicates the distance from the heat source centre. As the black curves indicate, the temperature rises sharply immediately after the heat excitation, and falls slowly after the peak. Also, the points closer to the heat source centre show a larger temperature rise and a more rapid temperature evolution. It should be noted that the temperature discussed in Fig. 5 and the following section is the temperature rise related to the initial one.

a) Titanium

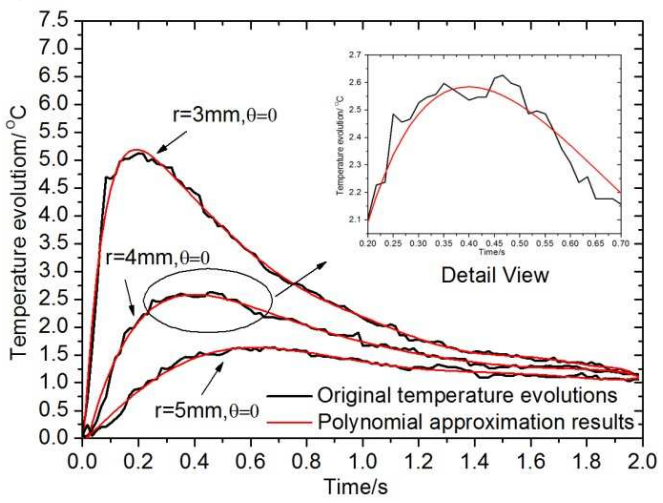

b) Zirconium

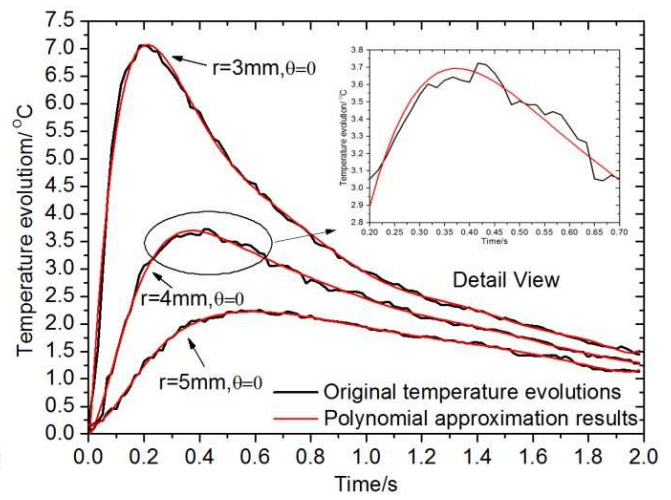

Fig. 5. The original temperature evolutions $T_{0}(r, t)$ and the polynomial approximation results $T_{p}(r, t)$.

To measure the thermal diffusivity, first the temperature variation rate should be calculated. As seen from the detailed pictures in Fig. 5, the original temperature evolutions $T_{0}(t)$ cannot be used to calculate the temperature rate due to the strong thermal noise. The 10 -order polynomial 
approximation is performed using (11), as described by the red curves in Fig. 5. The detailed pictures show that the smooth red curves without thermal noise indicating $T p(t)$ show a similar variation tendency to that of the black ones indicating $T_{0}(t)$. After performing the 10-order polynomial approximation processing on $T_{011}(t) \sim T_{0240320}(t), T p 11(t) \sim T p 240320(t)$ can be obtained. The temperature variation rate is calculated using (12), and the binary images of temperature variation rate are obtained by performing binarization processing combined with (13).

Figures $6 \mathrm{a}$ and $6 \mathrm{~b}$ show the image sequences of original temperature field, temperature variation rate, and temperature rate after binarization processing within the time range of $0.3 \mathrm{~s} \leq t \leq 1.2 \mathrm{~s}$, for the titanium and zirconium samples, respectively. The temperature field images display a visual temperature evolution process. They show that - soon after the heat excitation - a large temperature gradient is formed close to the heat source centre. As time increases, the sample temperature falls and tends to be the ambient temperature. The volumetric heat capacity of titanium $\left(c_{v}=2.34 \times 10^{6} \mathrm{Jm}^{-3} \mathrm{~K}^{-1}\right)$ is greater than that of zirconium $\left(c_{v}=1.81 \times\right.$ $\left.10^{6} \mathrm{Jm}^{-3} \mathrm{~K}^{-1}\right)$. Therefore, under the condition of the same heat excitation power $(25 \mathrm{~W})$ the temperature rise of titanium is lower than that of zirconium. Moreover, the thermal diffusivity of zirconium is larger than that of titanium, so the former will perform a faster thermal diffusion process. However, the difference between the two thermal diffusion processes cannot be distinguished clearly in the image sequences of original temperature field.

a) Titanium

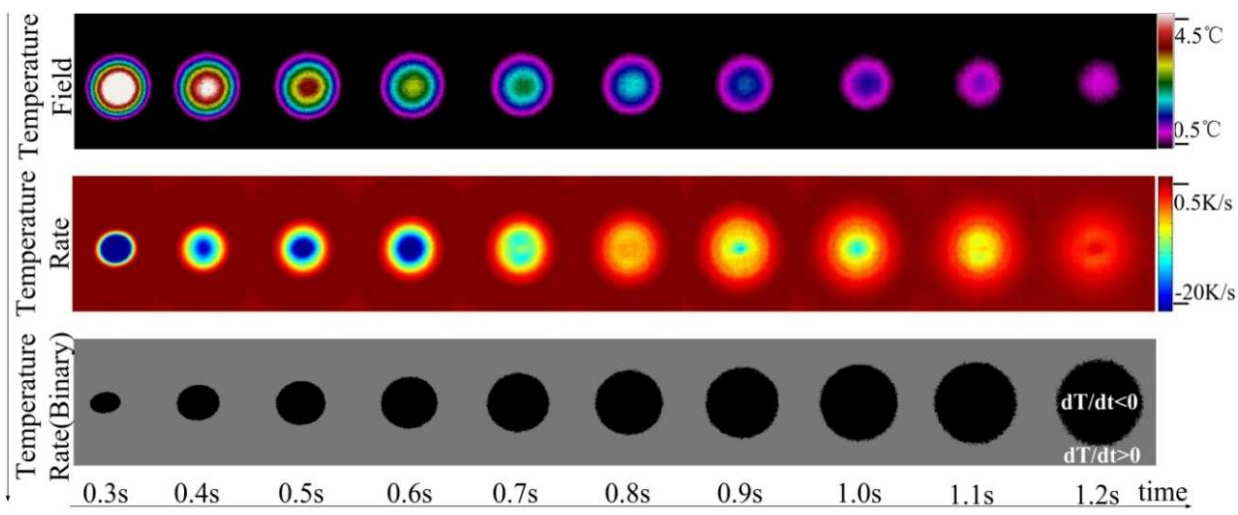

b) Zirconium

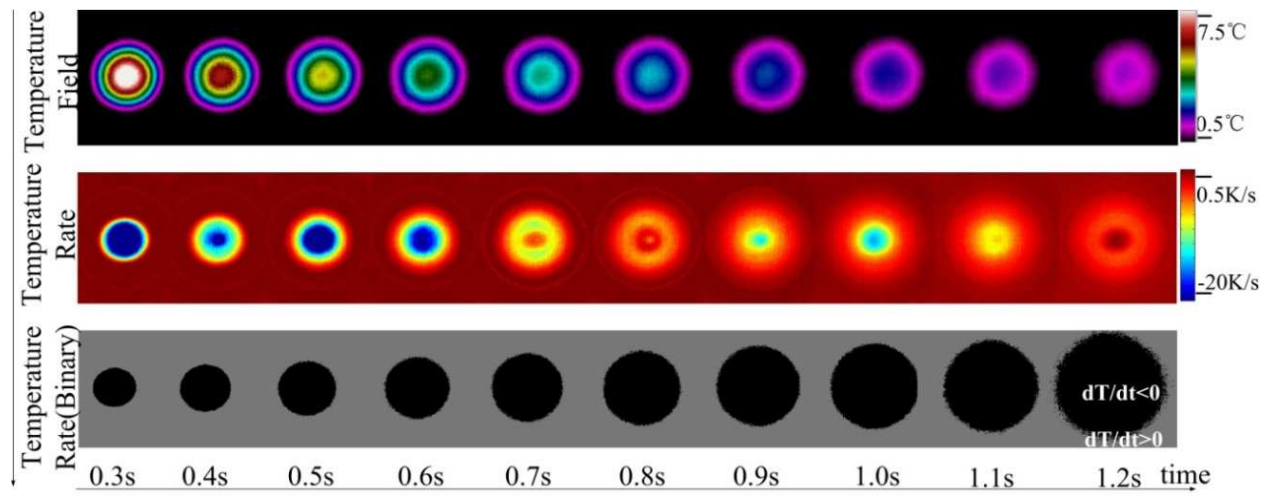

Fig. 6. The original temperature field images, images of temperature variation rate and binary images of temperature variation rate from $t=0.3 \mathrm{~s}$ to $t=1.2 \mathrm{~s}$. 
The image sequences of temperature rate are obtained by performing (12). We can see that the temperature variation rate is great at the early time. Then, it falls and tends to be zero with elapsing time. However, the thermal diffusion process cannot be observed clearly. Finally, binarization processing is performed on the image sequences of temperature rate using (13). Differently, the binary image frames of temperature variation rate describe the thermal diffusion process both clearly and quantitatively, as shown in Fig. 6 . At a specific time $t=t_{0}$, the temperature is falling $(d T / d t<0)$ in the black region, and rising $(d T / d t>0)$ in the grey region. Also, the area of the black region grows with time. It shows the same evolution process as the theoretically computed ones presented in Fig. 2. Besides, the black region of zirconium is larger than that of titanium at the same time. It is mainly due to the positive relationship between the thermal diffusivity value $\alpha$ and the area $s$ of the temperature falling region $(d T / d t<0)$, as shown in (9).

\subsection{DRLSE formulation and thermal diffusivity characterization}

Figure 7 shows $r_{\text {TMAX }}(\theta)$ calculation results obtained by performing DRLSE. In theory, the temperature falling and rising regions are segmented clearly by the maximum temperature curve $r_{\text {TMAX }}(\theta)$, as presented in Fig. 2. Although the binary image shows a rough contour, the $r_{\text {TMAX }}(\theta)$ cannot be determined directly due to the strong image noise around the contour, as shown in the left section of Fig. 7a. For the characteristic feature of fast convergence speed, good robustness and anti-noise capability [15] the DRLSE formulation is performed to segment binary images, and the calculated contour curves are considered as $r_{T M A X}(\theta)$. The yellow curve located between the two regions shows the calculated $r_{T M A X}(\theta)$, as shown in the right section of Fig. 7a. [15] has discussed the principle and application of DRLSE formulation in detail. This discussion will not be repeated here.

a) Detail view
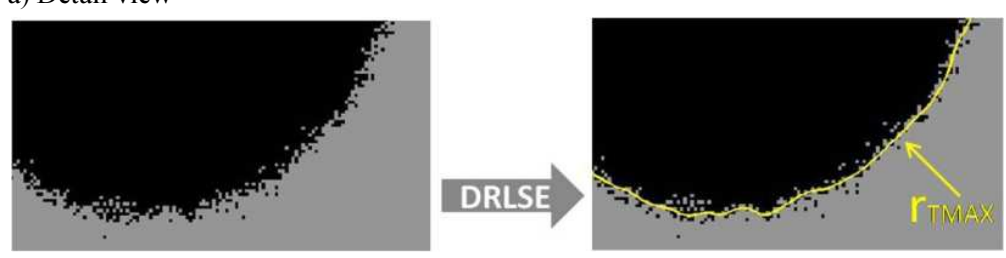

b) Titanium

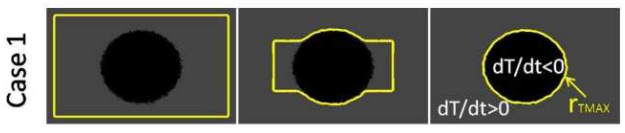

c) Zirconium
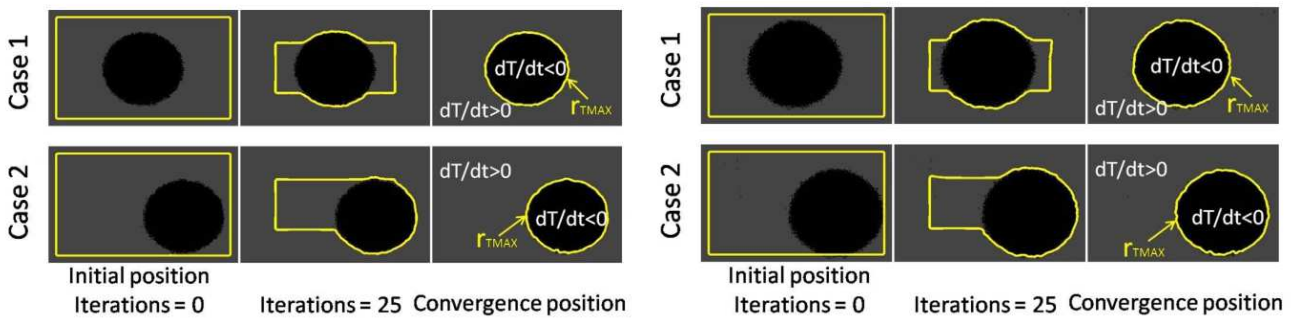

Fig. 7. The DRLSE formulation for the binary images at time $t=1.0 \mathrm{~s}$ for two typical measurement cases.

Figures $7 \mathrm{~b}$ and $7 \mathrm{c}$ show the DRLSE calculation process of the binary images at time $t=1.0 \mathrm{~s}$. Case 1 presents a typical setup for most existing laser pulse measurement methods, in which the laser beam centre is required to be aligned with the detection view centre of the IR camera. Differently, Case 2 shows a typical off-centre situation which might happen during on-line 
detections. The initially rectangular $r_{T M A X}(\theta)$ (the yellow curve) is set near the image edge, and it converges to the image contour with increasing iterations.

The convergence condition is given by $\left|\left(s_{q+1}-s_{q}\right) / s_{q}\right|<1 \times 10^{-4}$ ( $q$ represents the number of iterations), under which the final numbers of iterations are about 63 (Ti) and 58 (Zr) in Case1, and about $70(\mathrm{Ti})$ and $67(\mathrm{Zr})$ in Case 2 . It is clear that Case 2 indicating the typical off-centre situation obtains similar calculation results to those of Case 1, except for a small increase in the number of iterations.

The $r_{\text {TMAX }}(\theta)$ of all the 31 binary images of temperature variation rate within the time range of $0.5 \mathrm{~s} \leq t \leq 1.0 \mathrm{~s}$ are extracted by performing the DRLSE formulation. Then, the corresponding $\left(s_{1}, t_{1}\right) \sim\left(s_{31}, t_{31}\right)$ can be obtained. The measurements are repeated 12 times, the mean values $\overline{s_{1}} \sim \overline{s_{31}}$ with standard deviations are shown in Fig. 8. It shows that the standard deviations grow rapidly with increasing time. In particular, the standard deviations are $9.61 \times 10^{-7} \mathrm{~m}^{2}(\mathrm{Ti})$ and $8.87 \times 10^{-7} \mathrm{~m}^{2}(\mathrm{Zr})$ for $\overline{s_{1}}(t=0.5 \mathrm{~s})$, and $4.70 \times 10^{-6} \mathrm{~m}^{2}(\mathrm{Ti})$ and $4.04 \times 10^{-6} \mathrm{~m}^{2}(\mathrm{Zr})$ for $\overline{s_{31}}$ $(t=1.0 \mathrm{~s})$. To reduce the random error, the image sequences within the range of $t \leq 1.0 \mathrm{~s}$ are extracted for calculation. On the other hand, the number of sampling points of the IR camera will drop to less than 30 when $t<0.5 \mathrm{~s}$, which is not enough to calculate the temperature variation rate using (11) and (12). Given both concerns, the time range of $0.5 \mathrm{~s} \leq t \leq 1.0 \mathrm{~s}$ is determined for thermal diffusivity characterization. Linear regression is performed by inserting $\left(\overline{s_{1}}, t_{1}\right) \sim\left(\overline{s_{31}}, t_{31}\right)$ into (15). The fitting lines with corresponding correlation factors $r_{T i}{ }^{2} \approx 0.9998$ and $r_{Z r^{2}} \approx 0.9994$ for $\mathrm{Ti}$ and $\mathrm{Zr}$ samples, respectively, are shown in Fig. 8. The thermal diffusivity can then be characterized by substituting the fitting line slops $e_{T i}=1.167 \times 10^{-4}$ and $e_{Z r}=1.550 \times 10^{-4}$ into (16):

$$
\left\{\begin{array}{l}
\alpha_{T i}=1.167 \times 10^{-4} / 4 \pi=9.29 \times 10^{-6} \mathrm{~m}^{2} \mathrm{~s}^{-1} \\
\alpha_{Z r}=1.550 \times 10^{-4} / 4 \pi=1.23 \times 10^{-5} \mathrm{~m}^{2} \mathrm{~s}^{-1}
\end{array} .\right.
$$

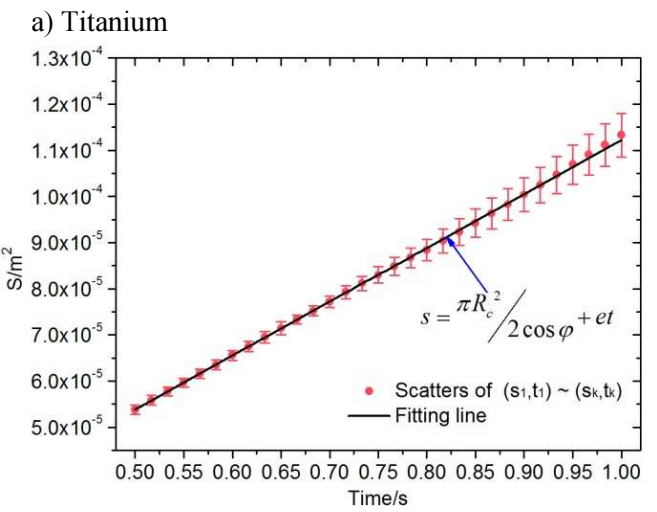

b) Zirconium

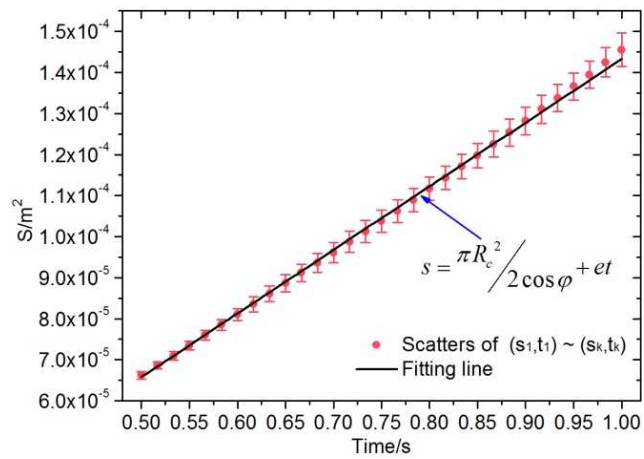

Fig. 8. The linear regressions combined with (15) for Ti and $\mathrm{Zr}$ samples.

\subsection{Measurement errors}

The samples of titanium, zirconium, and 304 stainless steel have been measured repeatedly. For each sample, 40 repeated measurements have been performed, and the random measurement errors with the limit error bands $\delta= \pm 3 \sigma(\delta$ is the limit random error, $\sigma$ is the standard deviation, and the corresponding confidence probability is $99.73 \%$ [20]) are shown in Fig. 9. As seen from Table 1, the relative deviations between the average measurements and 
the reference values [21] are: $-2.26 \%,-1.07 \%$ and $1.61 \%$, for 304 stainless steel, titanium, and zirconium samples, respectively.

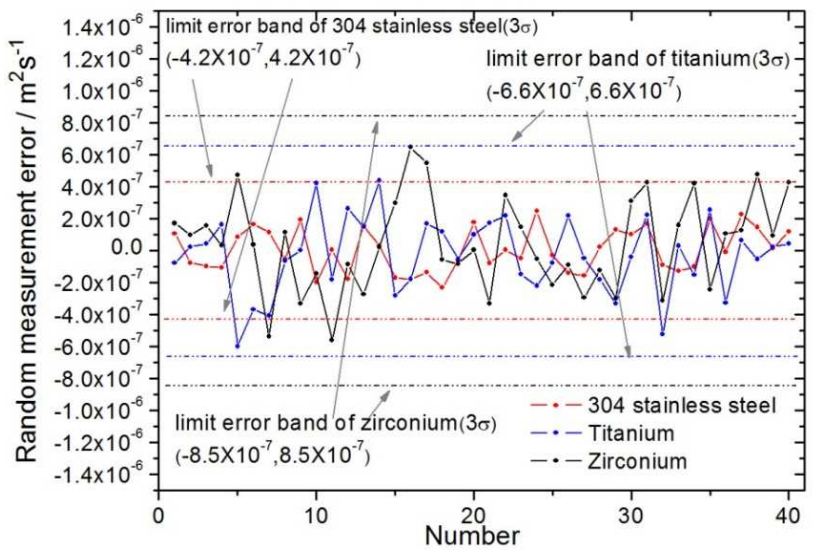

Fig. 9. The random measurement errors of the three samples.

Table 1. The measured thermal diffusivities of 304 stainless steel, titanium, and zirconium samples compared to the reference values.

\begin{tabular}{|c|c|c|c|}
\hline \multirow{2}{*}{ Sample } & \multicolumn{2}{|c|}{ Thermal diffusivity $\left[10^{-5} \mathrm{~m}^{2} \mathbf{s}^{-1}\right]$} & \multirow{2}{*}{$\begin{array}{c}\text { Deviation between the } \\
\text { measured value and } \\
\text { the reference }\end{array}$} \\
\cline { 2 - 3 } & Measured Value & $\begin{array}{c}\text { Reference } \\
\text { Value [21] }\end{array}$ & \\
\hline 304 stainless steel & $0.389 \pm 0.042$ & 0.398 & $-2.26 \%$ \\
\hline Titanium & $0.922 \pm 0.066$ & 0.932 & $-1.07 \%$ \\
\hline Zirconium & $1.26 \pm 0.09$ & 1.24 & $1.61 \%$ \\
\hline
\end{tabular}

\section{Conclusions}

This paper presents a novel method for simple and fast on-line detection of thermal diffusivity of thin films. The key feature of this method is that the off-centre detection error is avoided by performing the DRLSE formulation. The method is validated for three samples of 304 stainless steel, titanium, and zirconium using an on-line measurement system. The relative deviations between the mean measurement results and the references are less than $2.26 \%$.

Besides:

1. In our measurement system a laser power meter is introduced for laser energy metrology. A more portable system composed only of an IR camera, a laser source and a PC can be quickly built.

2. The data processing of a single measurement takes about 4 minutes (Intel Core i7-2640 CPU (a) $2.8 \mathrm{GHz}$ and $8 \mathrm{~GB}$ RAM for the PC, Matlab R2010b). Thus, it is possible to perform a fast on-line detection in the industrial conditions. 


\section{Acknowledgements}

This work was financially supported by National Basic Research Program of China (973 Program) (No. 2012CB934103).

\section{References}

[1] Suzuki, T., Kanno, I., Loverich, J.J., Kotera, H., Wasa, K. (2006). Characterization of Pb (Zr, Ti) $\mathrm{O}_{3}$ thin films deposited on stainless steel substrates by RF-magnetron sputtering for MEMS applications. Sensors and Actuators A: Physical, 125(2), 382-386.

[2] Kim, S.H., Leung, A., Koo, C.Y., Kuhn, L., Jiang, W., Kim, D.J., Kingon, A.I. (2012). Lead-free (Na.5 $\left.\mathrm{K}_{0.5}\right)\left(\mathrm{Nb}_{0.95} \mathrm{Ta}_{0.05}\right) \mathrm{O}_{3}-\mathrm{BiFeO}_{3}$ thin films for MEMS piezoelectric vibration energy harvesting devices. Materials Letters, 69, 24-26.

[3] Joost, U., Juganson, K., Visnapuu, M., Mortimer, M., Kahru, A., Nõmmiste, E., Ivask, A. (2015). Photocatalytic antibacterial activity of nano- $\mathrm{TiO}_{2}$ (anatase)-based thin films: Effects on Escherichia coli cells and fatty acids. Journal of Photochemistry and Photobiology B: Biology, 142, 178-185.

[4] Juez, R.G., Boffa, V., Blank, D.H., Johan, E. (2008). Preparation of self-supporting mesostructured silica thin film membranes as gateable interconnects for microfluidics. Journal of Membrane Science, 323(2), 347351 .

[5] Mostowfi, F., Czarnecki, J., Masliyah, J., Bhattacharjee, S. (2008). A microfluidic electrochemical detection technique for assessing stability of thin films and emulsions. Journal of colloid and interface science, 317(2), 593-603.

[6] Parker, W.J., Jenkins, R.J., Butler, C.P., Abbott, G.L. (1961). Flash method of determining thermal diffusivity, heat capacity, and thermal conductivity. Journal of applied physics, 32(9), 1679-1684.

[7] Cielo, P., Utracki, L.A., Lamontagne, M. (1986). Thermal-diffusivity measurements by the convergingthermal-wave technique. Canadian Journal of Physics, 64(9), 1172-1177.

[8] Cernuschi, F., Russo, A., Lorenzoni, L., Figari, A. (2001). In-plane thermal diffusivity evaluation by infrared thermography. Review of scientific instruments, 72(10), 3988-3995.

[9] Kim, S.W., Kim, J.C., Lee, S.H. (2006). Analysis of thermal diffusivity by parameter estimation in converging thermal-wave technique. International journal of heat and mass transfer, 49(3), 611-616.

[10] Murphy, F., Kehoe, T., Pietralla, M., Winfield, R., Floyd, L. (2005). Development of an algorithm to extract thermal diffusivity for the radial converging wave technique. International journal of heat and mass transfer, 48(7), 1395-1402.

[11] Kim, J.C., Kim, D.J., Kim, D.S., Kim, S.W., Troitsky, O.Y. (2001). One-level, two-point method for estimation of thermal diffusivity by the converging thermal-wave technique. International journal of thermophysics, 22(3), 933-942.

[12] Lu, G., Swann, W.T. (1991). Measurement of thermal diffusivity of polycrystalline diamond film by the converging thermal wave technique. Applied physics letters, 59(13), 1556-1558.

[13] Correa, P.R., Pereira, T.M., Veloso, M.N., Zezell, D.M. (2012) Development of a communication interface to determinate the thermal diffusivity as a function of temperature by infrared thermography. 11th International Conference on Quantitative Infrared Thermography, Naples, Italy.

[14] Joo, Y., Park, H., Chae, H.B., Lee, J.K., Baik, Y.J. (2001). Measurements of thermal diffusivity for thin slabs by a converging thermal wave technique. International journal of thermophysics, 22(2), 631-643.

[15] Li, C., Xu, C., Gui, C., Fox, M.D. (2010). Distance regularized level set evolution and its application to image segmentation. IEEE Transactions on Image Processing, 19(12), 3243-3254.

[16] Yang, F., Qin, W., Xie, Y., Wen, T., Gu, J. (2012). A shape-optimized framework for kidney segmentation in ultrasound images using NLTV denoising and DRLSE. Biomedical engineering online, 11(1), 82.

[17] Jin-qing, L., Wei-wei, L. (2011). Adaptive Medical Image Segmentation Algorithm Combined with DRLSE Model. Procedia Engineering, 15, 2634-2638. 
[18] Usha, R.N., Pv, S.D., Venkata, R.D.D., Nalini, K. (2011). Optimal Segmentation of Brain Tumors using DRLSE Levelset. International Journal of Computer Applications, 29(9), 6-11.

[19] Rianto, I., Pranowo, P. (2013). Distance Regularized Level Set Evolution for Medical Image Segmentation. The 1st Conference on Information Technology, Computer, and Electrical Engineering (CITACEE), 1, 4951.

[20] Chen, F.F. (2007) Fundamental Technology for Instrument Design. Beijing: Tsinghua University press, 424425.

[21] Incropera, F.P. (2011) Fundamentals of Heat and Mass Transfer. John Wiley \& Sons, Ltd, 905-908. 